\title{
SAND97-0255C
}

\section{A Simple Ionizing Radiation Spectrometer/Dosimeter based on Radiation Sensing Field Effect Transistors (RadFETs).}

\author{
D. J. Moreno, R. C. Hughes, M. W. Jenkins, and C. R. Drumm \\ Sandia National Laboratories, ABQ NM 87185, USA
}

\section{Summary}

This paper reports on the processing steps in a silicon foundry leading to improved performance of the Radiation Sensing Field Effect Transistor (RadFET) and the use of multiple RadFETs in a handheld, battery operated, combination spectrometer/dosimeter.

Keywords: Radiation, Dosimetry, Spectrometer

\section{Introduction}

The storage of large amounts of special nuclear materials and radioactive waste coupled with concerns about terrorism has led to a need for improved sensing of ionizing radiation. Our long term goal is to develop a RadFET technology that is compatible with silicon read-out electronics for a small, rugged integrated, dosimetry system for a wide range of doses and types of radiation.

The RadFET concept for measuring radiation dose has been around for many years [1]. It is based on the physics that $\mathrm{SiO}_{2}$ dielectric found in MOSFET gates shows high mobility electrons and low mobility holes when excited by ionizing radiation. The trapped holes provide a net positive charge which creates a permanent shift in the FET's threshold voltage.

The RadFETs discussed in this paper are based on our [2] dual dielectric design of $\mathrm{SiN}$ over $\mathrm{SiO}_{2}$. The RadFETs were fabricated at the Micrel Corp. in San Jose, CA. to specifications provided by the authors. Micrel was able to use their standard p-MOSFET process with the addition of a Low Pressure Chemical Vapor Deposition (LPCVD) SiN deposition after gate oxidation to fabricate the RadFETs. Micrel performs a high dose arsenic implant to provide a low resistance top-side contact to the (100) $\mathrm{n}$ on $\mathrm{n}+\mathrm{Si}$ epitaxial substrate. A $2.5 \mu \mathrm{m}, 1100^{\circ} \mathrm{C}$, wet oxidation and the negative photoresist are used to mask the implant whose beam current is less than $400 \mu \mathrm{A}$. The resist is removed by an $\mathrm{O}_{2}$ plasma followed by a wet chemical cleaning step. After a $1000^{\circ} \mathrm{C}$ arsenic drive-in and a source-drain masking operation, $\mathrm{BBr}_{3}$ is diffused at $1030^{\circ} \mathrm{C}$. A $1000^{\circ} \mathrm{C}$ field oxidation is performed to produce a field oxide of $12 \mu \mathrm{m}$ and a $\mathrm{p}+$ junction depth of $1.75 \mu \mathrm{m}$. After depositing and densifying an $8 \mu \mathrm{m}, 2.5 \%$ phosphorous CVD oxide, negative resist is used to etch the gate region followed by a wet chemical resist strip. A $875^{\circ} \mathrm{C}$, dry, sacrificial gate oxide is grown and etched before the gate oxidation and the nitride deposition.

There are a wide variety of applications for RadFETs and many of them require different electrical characteristics to fit in a system.

Sensitivity. The sensitivity of the RadFET is expressed in units of millivolts per rad dose. The shift in the FET is usually the same whether it is measured with the gate biased to give a threshold current, or at a higher current level where thermal effects are canceled out. Thus we refer to the "threshold voltage shift" even though the measurement is often made at the higher current level. Higher sensitivity is most often obtained by increasing the thickness of the $\mathrm{SiO}_{2}$ layer. We have fabricated RadFETs with a range of $\mathrm{SiO}_{2}$ thicknesses from 0.02 to 1 micron, giving sensitivities from 0.01 to $5 \mathrm{mv}$ per rad. Some applications (like the "Pocket Radiac" for soldiers) require that no battery can be used and thus zero gate bias during dose accumulation. The dose reader in this case is a separate instrument. The sensitivity is lower at zero bias, but because of electron diffusion in the $\mathrm{SiO}_{2}$ it can be surprisingly high: as much as $1 \mathrm{mv} / \mathrm{rad}$. Through a combination of processing steps including varying the nitride deposition, using $\mathrm{p}^{+}$polysilicon gates, and varying the gate oxide annealing process, we developed a new RadFET design with a zero bias performance that is comparable to the performance of a biased device.

Sensitivity at higher gate biases is voltage dependent because of geminate recombination effects; for best sensitivity in a given device a large gate bias during irradiation is desirable, but often that voltage will not be available in the system.

Initial Threshold Voltage: The most sensitive RadFETs with thick dielectrics will have large intial threshold voltages because of the normal doping levels of silicon used in the process. This means that the initial read voltages may be in excess of -10 volts, and as radiation dose accumulates the read voltage will be several volts larger. Many battery operated systems can not supply these voltages, so we conducted of study of the use of threshold voltage adjusting implants to put the initial threshold voltage near zero. We found it was possible to move the threshold voltage as much 


\section{DISCLAIMER}

This report was prepared as an account of work sponsored by an agency of the United States Government. Neither the United States Government nor any agency thereof, nor any of their employees, makes any warranty, express or implied, or assumes any legal liability or responsibility for the accuracy, completeness, or usefulness of any information, apparatus, product, or process disclosed, or represents that its use would not infringe privately owned rights. Reference herein to any specific commercial product, process, or service by trade name, trademark, manufacturer, or otherwise does not necessarily constitute or imply its endorsement, recommendation, or favoring by the United States Government or any agency thereof. The views and opinions of authors expressed herein do not necessarily state or reflect those of the United States Government or any agency thereof. 


\section{DISCLAMMIRR}

Portions of this document may be illegible in electronic image products. Images are produced from the best available original document. 
as 7 volts without impairing the sensitivity or drift characteristics of the RadFETs.

Read Current Levels: Our original RadFETs required several hundred microamps of read current at the point of zero temperature coefficient (ztc). This was deemed as too power hungry for several applications. We were able to reduce the read current levels at ztc below 50 microamps by redesigning the RadFET dimensions. Again we found that we did not have to sacrifice sensitivity or drift characteristics to achieve the low ztc devices.

Drift: Since the RadFET is a integrating device, the true "signal to noise" is different than that of a dose rate measuring device like a photodiode and is dominated by "drift". In many applications it will be necessary to have accurate readings of the accumulated dose over weeks or months, and so any changes in the threshold voltage independent of those caused by radiation are "drift" and degrade the accuracy of the reading. Our dual dielectric RadFET showed a marked decrease in drift characteristics over earlier single dielectric versions of the RadFET [2]. We attributed part of this to the presence of the negative gate bias during irradiation that pulls holes away from the critical Si$\mathrm{SiO}_{2}$ interface where interface state creation can cause a form of drift. Our best performing RadFETs now show postirradiation drift of about $5 \%$ of full scale reading over 1000 hours after the irradiation stopped. We have found that some processing steps lead to considerably higher drift, but we do not have a model for the microscopic origins of the drift.

\section{RadFET Array Spectrometer}

The RadFET has several advantages over photocurrent measuring devices like air ionization chambers and nuclear spectroscopy systems using semiconductor crystals. It stores the dose information without power and does not miss fast pulse radiation events; it requires only low voltages typical of silicon analog circuitry. Its main disadvantage is that it can not record individual $x$-ray photon events and has no inherent energy resolution. To extend the system performance we have designed, fabricated and tested a simple spectrometer/dosimeter system. The model-based RadFET array design consists of four elements with two metal filters for blocking low energy photons below two energy levels, a thin foil for amplifying the low energy photons through dose enhancement effects, and a bare (epoxy coated) device for measuring photons from the entire energy spectrum.

Several different radiation-transport codes were used in the analyses. The x-ray spectra were computed using the MCNP code[3]. MCNP is a general geometry, Monte Carlo radiation transport code. Given a geometric configuration and isotopic composition of some arrangement of special nuclear material (SNM), the MCNP code computes the distribution of photons emitted due the decay of the radioactive materials[4]. The code keeps track of the energy, spatial, and angular distribution of the emitted photons and accounts for the self shielding of the photons in the materials. The MCNP code determines the photon fluence at specified "detector" points surrounding the configuration of SNM.

The various filters were designed with a series of onedimensional photon-transport calculations. Two different codes were used in the analyses. The TIGER code of the Integrated Tiger Series (ITS) code package[5] is a Monte Carlo code that tracks photon and electron transport for onedimensional problem geometries. The CEPXS/ONEDANT code package[6] models electron and photon transport for one-dimensional geometries by the discrete ordinates (deterministic) method. The TIGER and CEPXS/ONEDANT codes both are able to calculate the distribution of energy deposited by photons passing through materials. The transport codes were used to compare various different filter materials and to determine the appropriate thicknesses for the applications. The TIGER and CEPXS/ONEDANT codes can generally be used interchangeably. The CEPXS/ONEDANT is somewhat more efficient for computing dose distributions, but the two codes provide a good check of each other.

The four RadFETs were designed into a printed circuit board with all the necessary analog read-out circuits including a voltage reference to tolerate varying battery voltage. The handheld unit shown in Fig. 1 also contains an ACR credit card sized data logger that records signal voltage from each RadFET at a predetermined time interval.

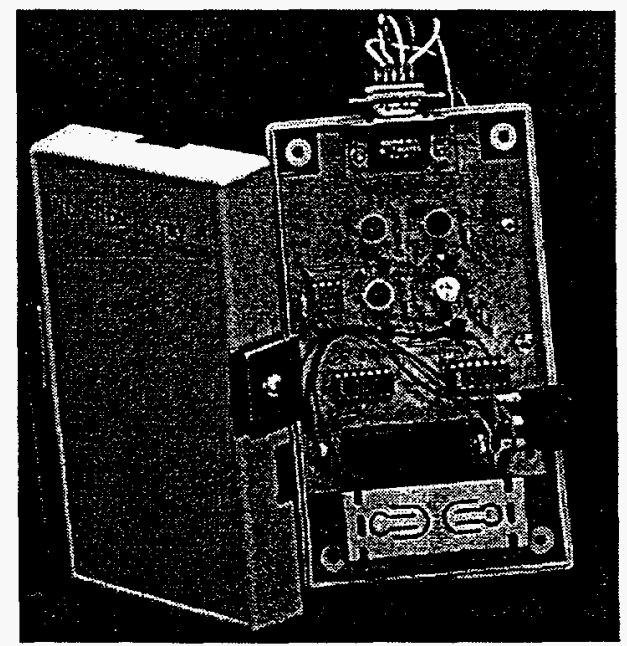

Fig. 1. The self-contained RadFET array spectrometer unit is contained in a 3 in. by 5 in plastic box. The four RadFETs in TO-5 headers can be seen in the top center of the printed circuit board. The filters are mounted inside the cover (not 
visible) and the ACR data logger is on the back. The unit can $\log$ data for about 3 months unattended on the enclosed batteries.

Fig. 2 shows the signals obtained from the handheld unit from a Np source over a period of 480 hours. The different signal levels from the RadFETs are consistent with the radiation transport code prediction for the $\mathrm{x}$-ray spectrum from $\mathrm{Np}^{237}$.

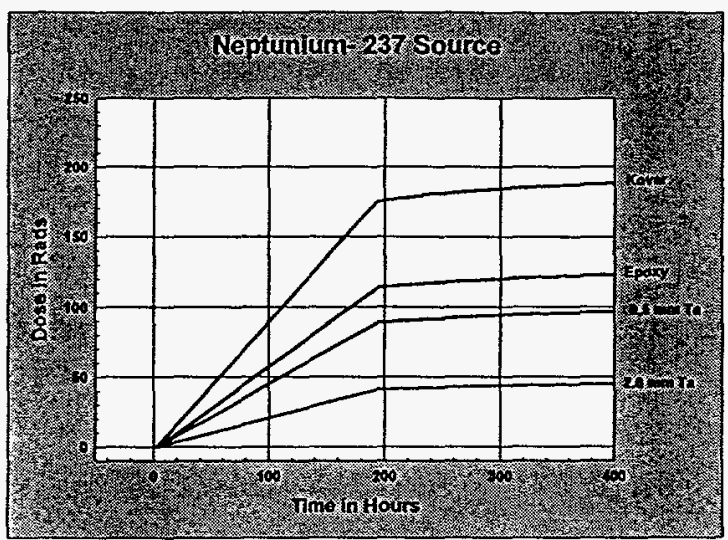

Fig. 2 Data from the spectrometer shown in Fig. 1 on exposure to a $\mathrm{Np}^{237}$ source. The source intensity at this position is about $0.8 \mathrm{rads} / \mathrm{hour}$. The filters in front of the four RadFETs are indicated at the right hand. This unit had previously received about 300 rads (epoxy) in prior experiments. The data points for this exposure were taken every 20 minutes and all values were set to zero at time zero, in addition to being corrected for temperature variations and converted from volts shift to rads. The spectrometer is removed from the source at 190 hours and then allowed to sit in a non-radiation environment for the rest of the data run.

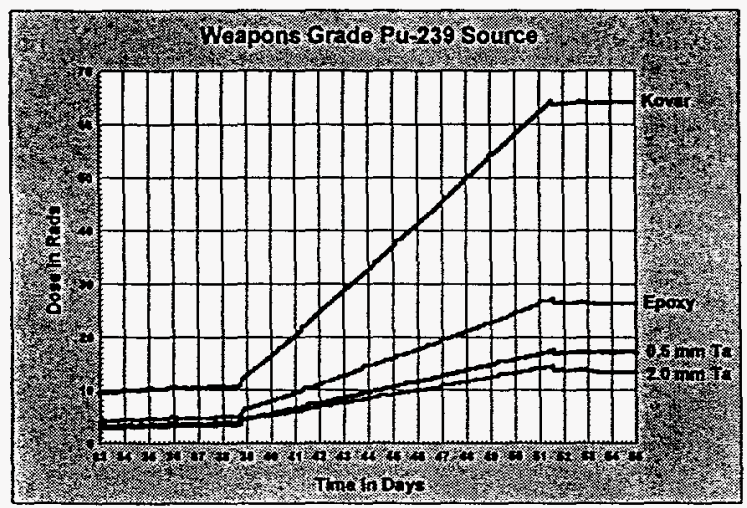

Fig. 3 The spectrum from a $P u^{239}$ source of old weapons grade $P u$ in a container. The dose rate at the container surface is about 70 millirads/hour. The unit was attached to the container by magnets. The initial dose received in an earlier exposure to the same source is not shown, but the accumulated dose after day 33 is shown. The unit was placed back on the source late in day 38 and removed in the middle of day 51. Each data point was corrected for the temperature at the time of reading from a temperature sensor in the $A C R$ data logger. Each voltage point was converted to rads by the simple ratio 10 millivolts/rad. The resolution of the unit is about 0.3 rads due to the 12 bit analog to digital converter in the ACR data logger.

Fig. 3 shows the signals obtained from a $\mathrm{Pu}^{239}$ source accumulated over 13 days. Fig. 4 shows a histogram of the ratio of responses relative to the bare (epoxy) RadFET. These ratios are the average of several sets of four signals taken at a given time like those in Figs. 2 and 3. As long as the total dose on the lowest signal is greater than about 5 rads, the ratios have less than $1 \%$ standard deviation. The histograms show that we can easily distinguish the four kinds of isotopes from one another with a simple pattern recognition program. The difference between the Np237 and old Pu239 was expected to be a challenge to the spectrometer because of similarity of their $\mathrm{x}$-ray spectra. The present filter set-up is predicted to also allows us to determine the age of $\mathrm{Pu}$ sources because of the change in $\mathrm{X}$-ray spectrum from aging.

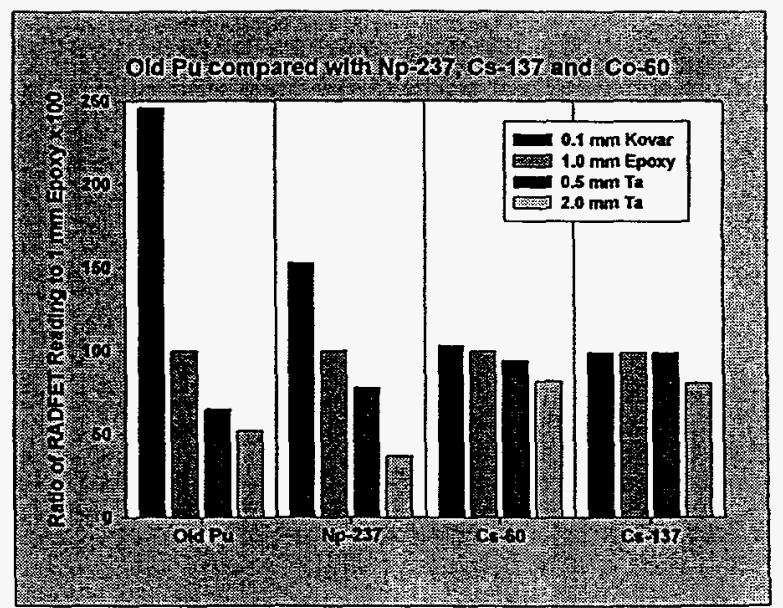

Fig. 4 Histogram of signals for four different isotopes.

\section{Conclusions}

In this paper we have reported on advances in the performance of the radiation sensing field effect transistor (RadFET) through process variations in Micrel's silicon fabrication facility. We have achieved improvements in zero 
bias response, initial threshold voltage, read current and drift over earlier RadFETs. We have assemble a self contained, battery operated RadFET array spectrometer for use in monitoring special nuclear materials. The energy resolution is sufficient to easier tell the difference between old Pu and $\mathrm{Np}^{237}$ isotopes in metal containers. These are also easily distinguishable from isotopes with much higher energy photons, like $\mathrm{Co}^{60}$ and $\mathrm{Cs}^{137}$. This low level of energy resolution may also be useful in applications where dose to humans from unspecified radiation sources need to be calculated; an example would be long space flights. Improvements in both sensitivity and stability will be necessary to fulfill the civilian personnel dosimetry market needs. There it would be required to read a one rad dose accumulation over a period of one year with about $20 \%$ accuracy. This market is currently be served by thermoluminescent dosimeters (TLDs). They require a very expensive $(\$ 50-90 \mathrm{~K})$ reader and can not give real time data.

\section{Acknowledgments}

We would like to acknowledge the assistance of Joe Cortez of the Los Alamos National Lab in obtaining the Pu data.

This work was supported by the United States Department of Energy under Contract DE-AC04-94AL85000. Sandia is a multiprogram laboratory operated by Sandia Corporation, a Lockheed Martin Company, for the United States Department of Energy.

\section{References:}

1. A. Holmes-Siedle and L. Adams, Radiat. Phys. Chem. $\underline{28}$, 235 (1986).

2. R. C. Hughes, W. R. Dawes, Jr., W. J. Meyer and S. W. Yoon, J. Appl. Phys. 65, 1972 (1989).

3. J. Breismeister (Editor), "MCNP-A General Monte Carlo N-Particle Transport Code, Version 4A," LA-12625-M, Los Alamos National Laboratory, Los Alamos, New Mexico (November 1993).

4. G. P. Estes, R. G. Schrandt, and J. T. Kriese, "Automated MCNP Photon Source Generation for Arbitrary Configurations of Radioactive Materials and First-Principles Calculations of Photon Detector Responses," LA-11153-MS, Los Alamos National Laboratory, Los Alamos, New Mexico (March 1988).

5. J. A. Halbleib, et al., "ITS Version 3.0: The Integrated TIGER Series of Coupled Electron/Photon Monte Carlo Transport Codes," SAND91-1634, Sandia National Laboratories, Albuquerque, New Mexico (March 1992).
6. L. J. Lorence, et al., "User's Guide to CEPXS/ONEDANT: A One-Dimensional Coupled Electron-Photon Discrete Ordinates Code Package, Version 1.0," SAND89-1661, Sandia National Laboratories, Albuquerque, New Mexico (September 1989). 